\title{
Design of Computerized Adaptive Testing Module into Our Dynamic Adaptive Hypermedia System
}

\author{
https://doi.org/10.3991/ijet.v16i18.23841 \\ Mohammed Boussakuk $^{1}\left({ }^{凶}\right)$, Ahmed Bouchboua ${ }^{1}$, Mohammed El Ghazi ${ }^{1}$, \\ Moulhime El Bekkali ${ }^{1}$, Mohammed Fattah ${ }^{2}$ \\ ${ }^{1}$ Sidi Mohamed Ben Abdellah University, Fez, Morocco \\ ${ }^{2}$ Moulay Ismail University, Meknes, Morocco \\ mohammed.boussakuk@usmba.ac.ma
}

\begin{abstract}
By the end of the 20th century, the most common and traditional paper and pencil tests (PBT) were faced with an increasing set of difficulties and drawbacks. Proceed and meet the measurement and evaluation needs of the $21 \mathrm{st}$ century; it is mandatory to have radical and qualitative changes. The accelerating pace of technological innovation in Education shows a clear path to computerbased testing, which offers a more engaging and innovative testing environment, as well as the ability to obtain instant results and feedback, the use of dynamic, multimedia learning objects (LO), and the implementation of adaptive, individualized assessment approaches. In this sense, this paper presents an adaptive formative assessment framework in which test adaptation is based on multiple criteria. The presented system bases its adaptation on the test takers' proficiency, schemata, desirable learning goal, cognitive level, and learning style. The main objective of this paper is to discuss the value of the adaptive testing system. Therefore, it presents the design and implementation of the system and the adaptive rules employed.
\end{abstract}

Keywords-Adaptive testing, e-Learning, Personalization, Learning Style, Intelligent Tutoring System.

\section{Introduction}

Nowadays, in this era of globalization, technological developments have opened up many electronic learning environment opportunities. As a result, technological advances have given rise to new approaches to learning and assessing. The latter (assessment) is a crucial factor in determining any learning system's success in evaluating learning outcomes. Assessment is an essential phase in the learning process that can influence the way of teaching and learning. In other words, the assessment provides helpful information to help the student identify what he/she has achieved and what he/she does not learn and his or her strengths, weaknesses, and misconceptions on the one hand. On the other hand, the assessment allows the teacher to observe the learning process's progress and, if necessary, intervene to adjust subsequent learning processes. 
For the reasons mentioned above, adaptive tests instead of classical tests are becoming a must.

Adaptive assessment systems are commonly referred to as Computerized Adaptive Testing (CAT) [1]. CAT is recognized as the next generation of assessments [2]. A CAT is a test delivered by a computer, where the selection of the questions given to the test candidates and the criterion to stop the test dynamically change according to the candidate's competence [3]. Computerized Adaptive Testing (CAT), in contrast to the traditional Paper and Pencil Test (PPT), is a process of test administration in which each student receives a tailored assessment based on his/her skills and abilities [1][3][4].

In the literature review, we found various studies discussing the benefits of CAT [1][5][6][7]. These studies' principal objective compares the measurement accuracy of the assessments and assess. The adaptive assessment aims at a reliable and effective measure of participant's knowledge level. The main advantage of CAT is that it provides short tests without presenting non-informative items to the candidate. That way, the adaptive assessment encourages candidates' motivation and avoids boring them by presenting tests appropriate to their knowledge level and competence. Thus, an examinee with a higher proficiency receives more difficult questions, unlike another examinee with lower proficiency. The CAT dynamically assesses and updates the candidate's estimated level of ability after each answer to a question in order to present the next question most appropriate to her/his ability level [1]. Each learner can start with different items, the learners do not receive the same subsets of items, and they can take different numbers of items from the item bank. More specifically, CATs are designed to adjust the difficulty of items to conform to the student's ability and skill.

In this paper, we first present our personalized and adaptive educational system called CleverUniversity, where each learner receives appropriate content according to her/his aptitudes, abilities, and learning style [8]. The current paper emphasizes presenting the adaptive formative assessment framework called CleverTesting, where the adaptation is based on a set of specific criteria, such as the competency, the pedagogical goals, the cognitive level, the needs, and the learning style of the test takers. Our system can be used for two purposes: as a test/items editor for teachers to build their tests with minimal effort and as an assessment environment for learners to take their formative tests online.

The remaining of the paper is organized as follows. Section 2 presents related scientific work on adaptive assessment and our contribution. The third section gives some information about different assessment strategies and introduces the Computerized Adaptive Testing process. Section 4 gives a general outline of the functioning of our adaptive e-learning system and its architecture. Section 5 introduces our proposed adaptive testing module, its architecture, and the different adaptation rules used. Finally, section 6 presents our conclusions and the future steps. 


\section{$2 \quad$ Related work}

Towards promoting and increasing adaptivity and individualization, many online learning tools have exploited the opportunities offered by adaptive testing. For example, SIETTE [9] is an automatic assessment environment for building and executing adaptive tests. The system supports different assessment theories, such as Classical Test Theory and Computer Adaptive Testing. Another study [10] presents an adaptive formative self-assessment framework that considers learners' learning style and their prior knowledge to customize the assessment task and provide effective specific feedback. In [11], the authors developed an English adaptive learning system (EALS) with a large item pool that accommodates varying difficulty levels for English students of different levels. As a result, each learner takes a personalized test adjusted to his or her proficiency level. Authors in [12] focus their research on mobile adaptive learning or context-aware mobile learning. They proposed an adaptive formative assessment approach whose main goal is to provide students with a personalized assessment taking into account their individual needs and contextual information. Furthermore, in [13], the authors present another approach to adaptive evaluation; the authors' method offers a new way for students to perform an individualized assessment using a multi-level hierarchical selection of items based on their difficulty and discrimination.

Other researchers [2][3][4][14] focus on Item Response Theory (IRT) and how it can contribute to improving the quality of computer-based adaptive assessment. More specifically, in [2], the authors proposed a framework for flexible deployment of CAT systems considering the following criteria: test domain, item selection strategy, and user interface. The prototype they implemented to demonstrate their approach's feasibility only generates tests consisting of dichotomous items evaluated with the One-Parameter Logistic model (1PL-IRT model). On the other hand, the authors in [4] developed an adaptive testing system to assess learners' performance using IRT with the Four-Parameter Logistic model (4PL). Also, in [3], the team studied the possibility of integrating an automatic question generation system (AQG) and a computerized adaptive testing (CAT) by predetermining the difficulty of the items without the need to administer them in a pretesting. In 2016, Hoang Tieu Binh [14] proposed to combine IRT and K-Means to assess learners' ability in an e-learning system to rank their level in 10 pre-defined groups. A similar system was introduced in [15], where the authors used the same machine learning approach, namely K-Means. After creating banks of items, they used the algorithm K-Means to obtain packages or sets of items with the same characteristics and the same level of difficulty in an automatic way. Other approaches in adaptive testing involve the use of fuzzy logic to improve the individualization and adaptivity of electronic assessment. For example, in [16], the authors presented a framework for creating adaptive tests based on the combination of Bloom's revised taxonomy cognitive theory and fuzzy logic to provide learners with distinct test items based on their abilities and learning requirements.

The most important conclusion from the literature review is that adaptive testing is an active topic for researchers. The proposed techniques aim at improving the efficiency of adaptive tests. However, most of these studies are based on the same principle: building adaptive items according to their level of difficulty and the student's level 
of knowledge, the better the proficiency, the more challenging the next question will be. Therefore, we present an adaptive formative assessment system that takes into consideration various criteria, such as the proficiency, the prior knowledge and the learning style of the learner, the type of exercises, and the pedagogical objectives according to the cognitive levels defined in Bloom's Taxonomy of Educational [17]. Our solution will help teachers understand their learners better and regulate the learning process according to their progress to the set goals. Tests and items are coded using the IMSQuestion and Test Interoperability v2.2 specification (IMS-QTI) to ensure interoperability and reuse between various compliant systems [18][19].

\section{Theoretical background}

\subsection{Assessment strategies}

Evaluation is central to teaching and learning. What is evaluated defines how it is learned and how it is taught. According to [20] and [21], three types of assessments can be found in the educational system: formative, summative, and diagnostic.

Formative assessment. Formative assessment (also known as assessments for learning) is a process that provides constructive feedback to both student and teacher on a given item or topic. This feedback helps teachers to detect the comprehension level, the weak and strong points of each learner. As such, it is usually carried out during the learning process. By exploiting the feedback, the students can understand the nature of their mistakes and the difficulties they encounter during the learning process. On the other hand, this type of assessment allows the instructional program to modify the progression rhythm to bring eventual improvements or corrections to achieve the set pedagogical objectives [22].

Summative assessment. Summative assessment (also known as assessment of learning): the primary purpose of this type of assessment is a certification goal or a judgment on the learner's overall performance [23]. Indeed, the summative assessment allows the recording, and often grading, of the student's achievement to the learning objectives set out in the curriculum. In other words, it is an indicator of the skills acquired. It takes place at the end of a learning process or course and provides a quantitative appreciation, and measures the candidate's progress. Summative assessment enables institutions to make decisions, classify learners to each other within a group, and enable students to obtain diplomas to move on to a higher level [21].

Diagnostic assessment. Diagnostic assessment (also known as pre-assessments) identifies learners' needs, skills, prior knowledge, and preconceptions to guide them towards the most appropriate learning program [23]. Generally undertaken at the beginning of a course, a diagnostic assessment should assess the current student's achievement across all relevant abilities, knowledge, and understanding. This type of assessment measures each learner's prerequisites, allowing the teacher to have a clear vision of the students' level of skills.

Table 1 presents the main differences between the three types of assessment. 
Paper-Design of Computerized Adaptive Testing Module into Our Dynamic Adaptive Hypermedia ...

Table 1. Summary of assessment types.

\begin{tabular}{|c|l|l|l|}
\hline & \multicolumn{1}{|c|}{ Diagnostic assessment } & Formative assessment & Summative assessment \\
\hline Problem & What do you know? & What are you learning? & What have you learned? \\
\hline Function & $\begin{array}{l}\text { Identify prior knowledge, skills, and } \\
\text { misconceptions to provide adequate } \\
\text { learning. }\end{array}$ & $\begin{array}{l}\text { Provide feedback and } \\
\text { monitor instructions. Help } \\
\text { teacher decision-making }\end{array}$ & $\begin{array}{l}\text { Assess learner learning } \\
\text { against some standard or } \\
\text { benchmark }\end{array}$ \\
\hline Timing & $\begin{array}{l}\text { At the beginning of the course, or } \\
\text { when a learner is having difficulties. }\end{array}$ & $\begin{array}{l}\text { During the instruction pro- } \\
\text { cess }\end{array}$ & $\begin{array}{l}\text { End of unit or course, } \\
\text { grading period }\end{array}$ \\
\hline Examples & Pre-assessments, observations & $\begin{array}{l}\text { Quizzes, homework, as- } \\
\text { signments, group work }\end{array}$ & $\begin{array}{l}\text { Final exams; final pro- } \\
\text { ject; term paper }\end{array}$ \\
\hline
\end{tabular}

Today, technology has greatly facilitated the production of assessments, but these tests offer all candidates' exact content regardless of the differences in their proficiency. For this reason, we have chosen to design an adaptive test production tool using open specifications to help students assess themselves before an exam.

The following section deals with a theoretical background of the adaptive assessment systems.

\subsection{Computerized Adaptive Test process}

Computerized Adaptive Testing (CAT) is an area of research emerging from psychometrics that has been adopted in recent years for summative and formative assessment tasks in e-learning environments. Unlike linear tests, CAT works by adapting a test to each candidate, question by question, according to their answers and ability level. If the candidate answers a question correctly, the next question will be more challenging to get as close as possible to his/her level of competence and vice versa [3]. The process of administering CAT is illustrated in figure 1 and has the following steps [1][3][24]:

- The test starts with estimating the initial ability level of the candidate. Then, the computer selects from the item bank the most appropriate item based on the test taker's current proficiency.

- The selected question is presented to the test taker and answered.

- Based on the response, the candidate's current proficiency is re-estimated to determine the next optimal item. Generally, if the candidate gives the correct answer, the next item will be slightly more complicated than the previous one. On the contrary, if the answer is incorrect, the next item will be more accessible.

- The last step is to check if the termination criterion has been reached. If not, the computer selects, administers another item, and the knowledge level is recalculated. This process is repeated until it reaches a specific termination criterion. 


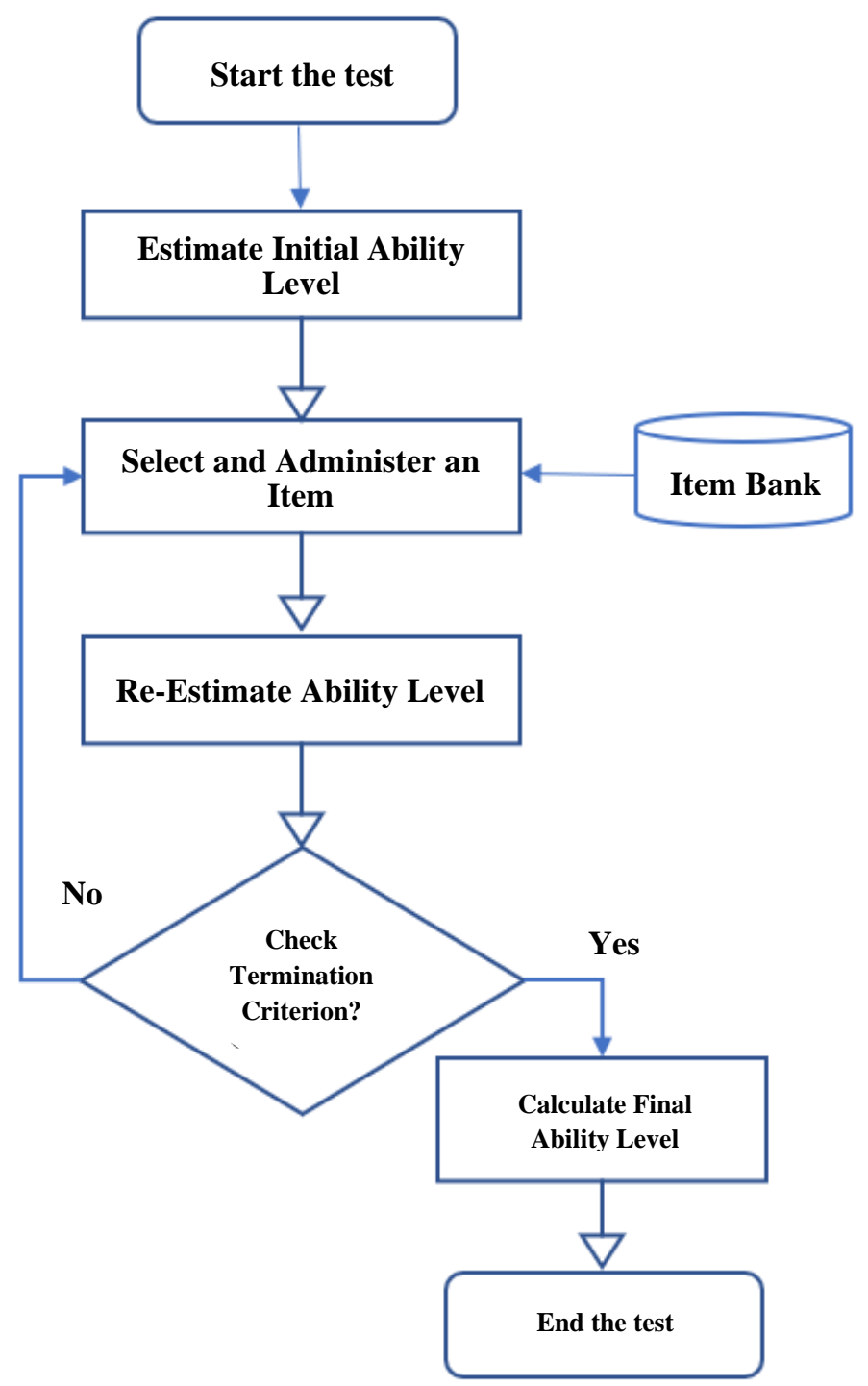

Fig. 1. Flow diagram of Computerized Adaptive Testing (CAT)

In this way, a CAT procedure consists of four main components: (a) an item pool, (b) an item selection procedure, (c) an ability estimation method, and (d) a termination criterion [1][25]. We note that there are various options for each of these elements in deploying CATs for a particular purpose [7][26].

- Item pool: or item bank, is a collection of test items with a full range of ability levels. The key to the success of any adaptive assessment system depends mainly on the item bank's quality. 
- Item selection procedure: CAT selects the following item to be asked according to the candidate's estimated level of competence. This improves accuracy and reduces the length of the test.

- Ability estimation method: Most CATs utilize Maximum Likelihood Estimation (MLE) or Bayesian methods to estimate the trait level of the examinee[26][14].

- Termination criterion: In a CAT system, the assessment continues only as long as necessary for each test taker. To this end, several rules can be used to determine the end of the assessment, depending on the test's objective. For example, the computer can end a test if the set number of items is reached or when the learner's level of knowledge is estimated to be at a statistically acceptable level.

To sum up, there are various components and methods for determining the process of an adaptive test. These elements can be combined in different ways, depending on the purpose of the test.

\section{Outlines of our DAHS}

Our team has implemented in previous works a Dynamic Adaptive Hypermedia System (DAHS) called "CleverUniversity" [8]. This is a personalized and adaptive educational environment where each learner receives appropriate content according to her/his aptitudes, abilities, and learning style. CleverUniversity is an individualized framework based on Felder\&Silverman learning style model (FSLSM). According to a recent survey conducted by [27], the FSLSM is the most frequent model adopted by many intelligent tutoring systems (ITS). FSLSM classifies students' learning styles (LS) in four dimensions: D1: active/reflective (Act/Ref), D2:sensing/intuitive (Sen/Int), D3:visual/verbal (Vis/Ver), and D4:sequential/global (Seq/Glo) [27][28]. The first time learners use CleverUniversity, they answer a questionnaire called Index of Learning Styles (ILS) that contains 44 questions to define their preferred learning style. Therefore, education content is generated for the learner according to her/his learning style. The individualization characteristic of our DHAS derives from this aspect. In other words, thanks to our intelligent system, each student receives educational resources tailored to their individual differences. This expert system stores all the learners' actions and traces to guide them in their learning process and offer them individualized tutoring. By personalizing learning paths, our system encourages learner motivation and avoids disorientation and cognitive overload in learning. Briefly, CleverUniversity supplies what student needs.

Figure 2 shows the logical architecture of our DHAS. The adaptation model is the main component responsible for adapting the content. Applying pedagogical rules can select the appropriate educational concepts according to the learners' specific characteristics, such as their learning style and knowledge level [8]. The learner module presents the core of any individualized learning by exploiting different information collected about the student, e.g., personal data, knowledge level, pedagogical objectives, preferred learning style. 


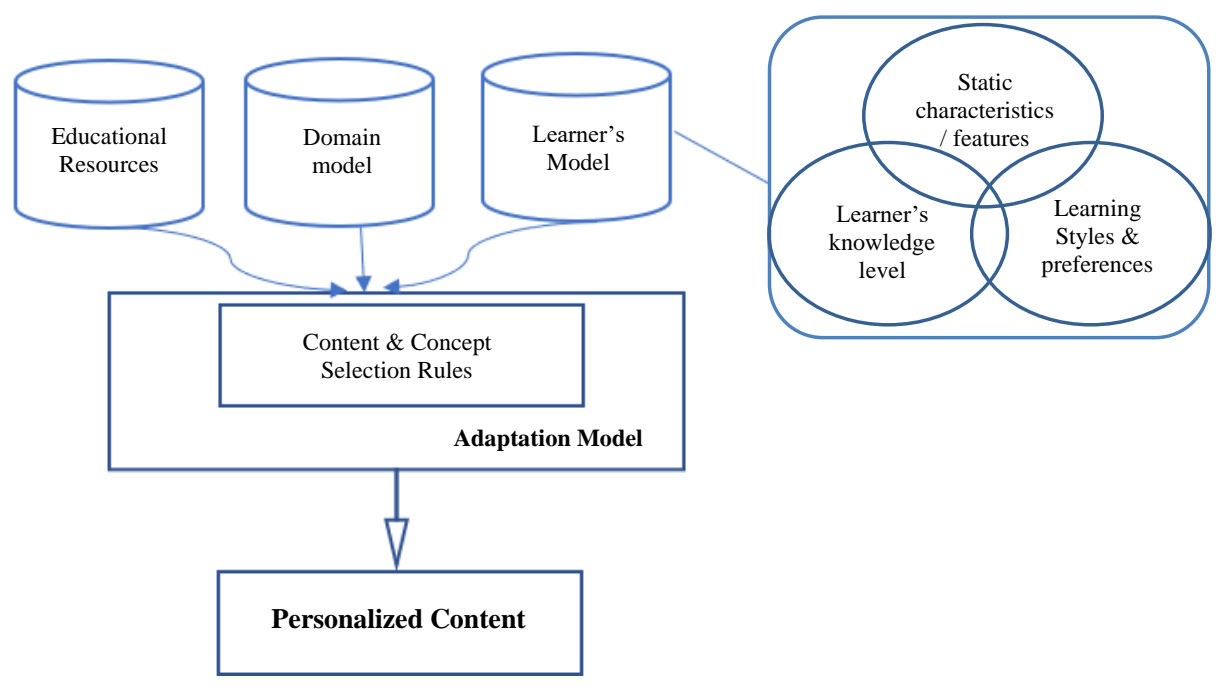

Fig. 2. Logical architecture of CleverUniversity.

We have paid particular attention to implementing flexible and user-friendly interfaces to develop our e-learning system, which is based on a three-tier architecture. A 3tier architecture is a well-established software application architecture that separates applications into three logical and physical layers: the presentation layer, the business logic layer, and the data layer. Our system is based on open technologies. It was developed in Laravel framework v.8, Vue.js v.3, Bootstrap v.4, Sass. MySql was used for the database.

\section{Adaptive assessment module in our DAHS}

\subsection{Architecture}

In this section, the architecture of our adaptive testing system, CleverTesting, is discussed in detail. The basic architecture of the CleverTesting module is shown in figure 3. As is seen in this figure, our system is composed of the following components: 


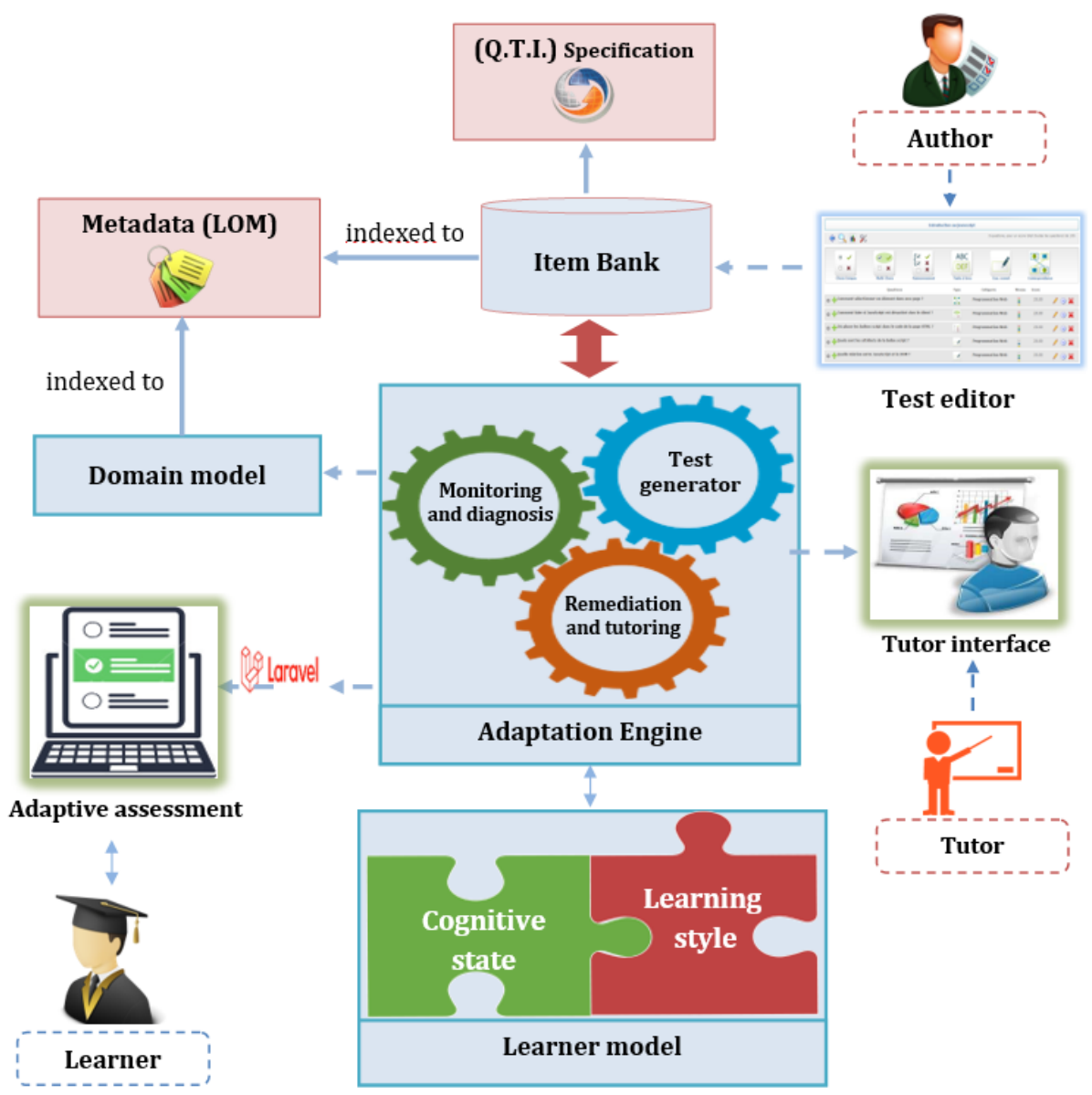

Fig. 3. Architecture of the proposed adaptive testing system.

- Learner model: This component maintains data about the learner's profile. Some of the information is introduced manually by the learner or the teacher, namely the learner's identification information, preferences, demographics, pedagogical objectives, and previous knowledge on specific topics. On the other hand, this module also handles dynamic learner data updated during the learner's interaction with the system, namely the learner's knowledge level, errors categories, learning style, skills, performance, results, and test activity data. All of these characteristics are required as the basis for designing and building adaptive testing.

In general, the student may prefer one learning style for one activity and another style for a different activity. Thus, our adaptive system is designed to identify each student's preferred learning style continuously and automatically.

- Domain model: maintains the different learning objects (concepts, questions, exercises, etc.) used in learning created at the most satisfactory level of granularity to ensure reusability regardless of the format used to create them. A structure with 
nodes and relationships is generally used. Many researchers use ontologies to build this educative organization [29].

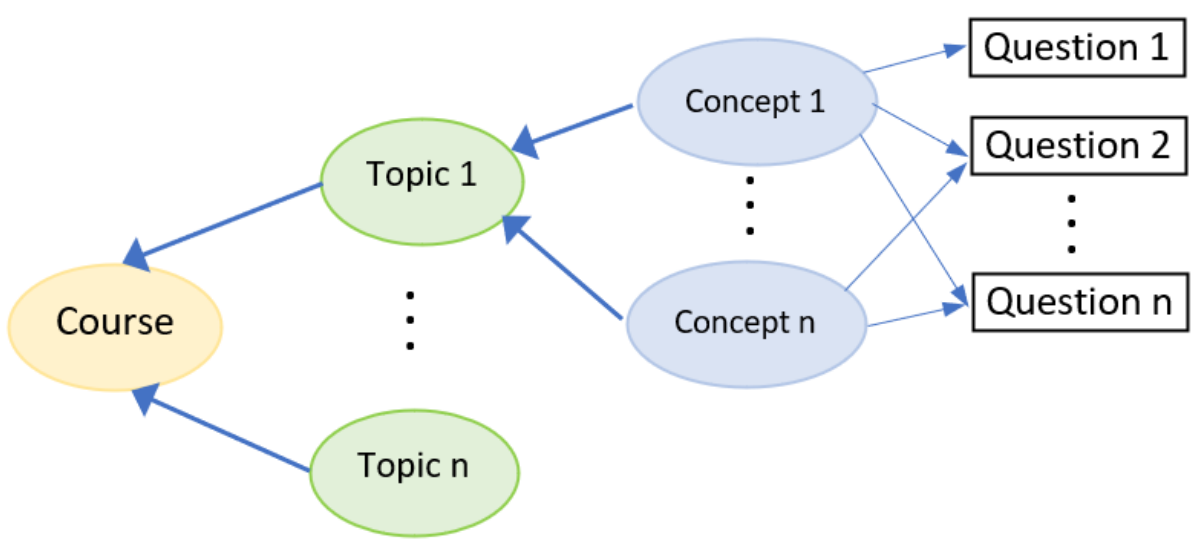

Fig. 4. Learning objects structure.

In the presented adaptive testing framework, exercise, test, and activity questions are associated with concepts. As shown in figure 4 , the instructional content is organized by topics, concepts, and sub-concepts. This hierarchical structure allows our system to relate test items to particular concepts. In other words, at the end of the assessment activity, the system updates the learner's model with the estimated knowledge level for the concept related to that activity. This is important from a pedagogical standpoint because it will help link learners' knowledge to specific concepts.

- Adaptation engine: is the main component of our system that performs the adaptation by applying a collection of item selection rules. This module receives as input the test items and their associated concepts, the learner's profile data, the rules and delivers the tests in adaptive mode to the test takers. Once the candidate finishes the test, the adaptation module updates his or her proper profile with the new knowledge estimate and presents reports on the tests taken. It also attempts to provide personalized support and feedback to the learner. These deductions are beneficial to both instructors and students to help them appreciate their accurate skills. For this purpose, the adaptive assessment module consists of three sub-modules: a tutoring module, a diagnostic \& monitoring module, and an exercise generator.

- Test Editor: is a web application based on a client/server architecture that the user can access via a web navigator. Our authoring application provides an intuitive and user-friendly interface to simplify the design of items and tests (figure 5), thus avoiding the technical complications behind the IMS-QTI format [18]. In more detail, the educator can use this tool to generate new items based on the IMS-QTI specification or choose them from the item bank. These assessment objects can be downloaded, reused, and swapped in an e-learning network. Furthermore, this editor provides import/export items and tests that conform to IMS-QTI standards. Besides, as seen in figure 5, authors can specify other supplementary parameters, such as test time limit, 
instructions to candidates, feedback, navigation mode, etc. These metadata correspond to the IMS-QTI specification's specific elements and are helpful to adapt the testing to the pedagogical objectives better. The educator can further enrich the questions with multimedia content, such as sounds, pictures, or videos. These multimedia elements are stored as separate files to be referenced and shared between different queries.

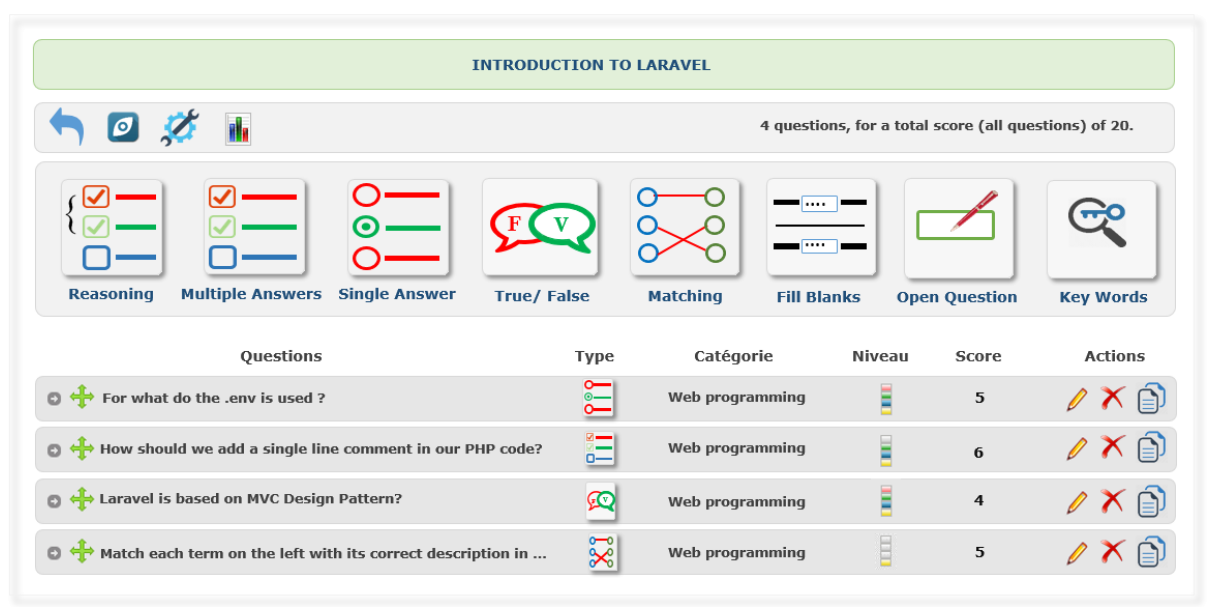

Fig. 5. The teacher creates a test via a user-friendly editor

Our new "CleverTesting" adaptive testing module will be integrated into our adaptive hypermedia system while considering its integration into other community projects like Moodle. Experiments will follow this phase to improve our platform and evaluate its effectiveness.

\subsection{Adaptation criteria}

As mentioned above, our adaptive tool considers several characteristics of the learners and the desired learning objective, and the type of questions. These criteria are the following:

- Level of knowledge: It is a crucial criterion for our adaptive assessment system to determine which test items are most appropriate for the candidate's level of knowledge. The adaptation engine analyzes the concepts' values to decide which ones are in the known and unknown range.

- Prior knowledge (schemata): A learner's prior knowledge of specific subjects can influence their level of knowledge or difficulty level of the items, which should be involved in the adaptive assessment. This parameter's value can be indicated as none, low, medium, or very good when initializing a new learner profile. Subsequently, the system updates the learner's knowledge level during his interaction with his learning environment. 
- Learning style: As mentioned in section 4, CleverUniversity is an individualized framework based on Felder\&Silverman learning style model (FSLSM). Assessment activities are generated according to the order of preference of the candidate's learning style. For example, exercises that contain pictures or graphics will be recommended for candidates with a visual learning style. On the other hand, for a learner with an auditory learning style, the exercises delivered to him/her should have a transcribed pronunciation. It is important to note that a learner's learning style may differ from one task to another. Therefore, an intelligent adaptive system must identify the most appropriate learning style for each learner.

- Revised Bloom Taxonomy (RBL) level: The RBT framework is used to categorize statements about what we anticipate or intend learners to learn due to instruction. The framework was designed to facilitate the exchange of test items between educational content producers to create item pools that all measure the same educational objective. This taxonomy helps create adaptive tests. According to RBT, cognitive objectives are organized into six levels; the first level concerns concepts that are easy to assimilate with a small degree of reasoning, then the concepts move up the level of knowledge to complex levels. These six levels are: Remembering, Understanding, Applying, Analyzing, Evaluating, and Creating [17]. The structure of the cognitive process dimension of the RBT is shown in table 2 .

- Preferences: Learners' preferences relate to the execution environment and its visual appearance. Graphic design that is reminiscent of the learning domain creates cognitive and affective imprinting that helps the candidate focus on the task. Thus, preferences such as font, colors, images, etc. could be included in the system to make navigation pleasant and support the assessment activity.

Table 2. The structure of the cognitive process dimension of the RBT

\begin{tabular}{|c|c|c|}
\hline RBT level & Characteristics of the level & Examples of action verbs \\
\hline Remember & $\begin{array}{l}\text { Recall and Memorize definitions. } \\
\text { Know the basic notions, formulas, and rules. }\end{array}$ & $\begin{array}{l}\text { Define, memorize, list, label, } \\
\text { recite }\end{array}$ \\
\hline Understand & $\begin{array}{l}\text { Interpret information based on what has been learned. } \\
\text { Explain or translate facts, procedures, etc. }\end{array}$ & $\begin{array}{l}\text { Classify, identify, describe, } \\
\text { explain, interpret }\end{array}$ \\
\hline Apply & $\begin{array}{l}\text { Use acquired skills and knowledge to solve problems. } \\
\text { Apply methods and/or theories in new situations. }\end{array}$ & $\begin{array}{l}\text { Apply, demonstrate, solve, } \\
\text { use, execute }\end{array}$ \\
\hline Analyze & $\begin{array}{l}\text { Distinguish, compare, and relate facts and structure of a } \\
\text { task or issue. } \\
\text { Detect the parts of material and distinguish how they are re- } \\
\text { lated to each other. }\end{array}$ & $\begin{array}{l}\text { differentiate, analyze, con- } \\
\text { trast, decompose, organize }\end{array}$ \\
\hline Evaluate & Make judgments based on criteria and standards. & $\begin{array}{l}\text { defend, judge, justify, meas- } \\
\text { ure, validate }\end{array}$ \\
\hline Create & $\begin{array}{l}\text { Generate new ideas or solutions } \\
\text { Plan strategies. }\end{array}$ & $\begin{array}{l}\text { Design, formulate, combine, } \\
\text { construct }\end{array}$ \\
\hline
\end{tabular}

The presented system creates adaptive tests according to the learning objective, which implies the learner's knowledge level, prior knowledge, learning style, and preferences. These tests include simple "single choice" questions to assess level 1, such as open-ended questions to assess more complex levels, which require solving a difficult 
problem or generating new methods and solutions. This system should select the most appropriate and relevant test item each time, based on the different criteria mentioned above, to provide personalized assessment activities that aim at more effective educational outcome.

\section{Conclusion}

This paper presents the components and processes of creating an adaptive hypermedia assessment system in which adaptation criteria can be defined to meet different pedagogical objectives. The objective of this work was to propose an adaptive testing framework based on multiple learner criteria. Student data is saved in individual profiles to adjust the teaching process according to the learner's characteristics. The data stored in the student profiles vary from demographic data to learning style, knowledge on specific subjects, and user objectives on the one hand. On the other hand, each question is described by several data and metadata such as the title of the question, description, possible answers, type, level of difficulty, educational objective. Based on these data, the system can apply different criteria to generate personalized tests for each learner.

This test personalization method's significant advantage is the degree of adaptability it offers to students and instructors. Instructors can draw on their pedagogical experience to create tests that are better adjusted to the characteristics of their students and provide additional tutoring as needed. Learners can build on prior knowledge or current objectives and are assessed by shorter tests with more focused items.

Our current work focuses on developing our adaptive assessment system; future work will be evaluated via experimental studies in a real classroom in a specific domain.

\section{$7 \quad$ References}

[1] D. J. Weiss and G. G. Kingsbury, "Application of Computerized Adaptive Testing to Educational Problems,” J. Educ. Meas., vol. 21, no. 4, pp. 361-375, 1984.

[2] S. Oppl, F. Reisinger, A. Eckmaier, and C. Helm, "A flexible online platform for computerized adaptive testing,” Int. J. Educ. Technol. High. Educ., vol. 14, no. 1, 2017. https://doi. org/10.1186/s41239-017-0039-0

[3] Y. Susanti, T. Tokunaga, and H. Nishikawa, "Integrating automatic question generation with computerised adaptive test," Res. Pract. Technol. Enhanc. Learn., vol. 15, no. 1, 2020. https://doi.org/10.1186/s41039-020-00132-w

[4] L. S. Riza, N. S. Nurjanah, and Y. Wihardi, "The development of computer adaptive test and item response theory with 4 parameters based the logistics model," J. Phys. Conf. Ser., vol. 1280, no. 3, pp. 1-6, 2019. https://doi.org/10.1088/1742-6596/1280/3/032033.

[5] H. Özyurt, Ö. Özyurt, A. Baki, and B. Güven, "Integrating computerized adaptive testing into UZWEBMAT: Implementation of individualized assessment module in an e-learning system," Expert Syst. Appl., vol. 39, no. 10, pp. 9837-9847, 2012. https://doi.org/ 10.1016/j.eswa.2012.02.168 
Paper-Design of Computerized Adaptive Testing Module into Our Dynamic Adaptive Hypermedia ...

[6] A. J. B. Pramono and H. Retnawati, "Implementation of cat in Indonesia school: Current challenges and strategies,” Univers. J. Educ. Res., vol. 8, no. 11, pp. 5599-5609, 2020. https://doi.org/10.13189/ujer.2020.081164

[7] E. Georgiadou, E. Triantafillou, and A. A. Economides, "Evaluation parameters for computer-adaptive testing," Br. J. Educ. Technol., vol. 37, no. 2, pp. 261-278, 2006. https://doi.org/10.1111/j.1467-8535.2005.00525.x

[8] M. Boussakuk, A. Bouchboua, M. EL-Ghazi, M. Fattah, and M. El-Bekkali, “A Fully individualized Adaptive and Intelligent Educational Hypermedia System : Details of CleverUniversity," Int. J. Emerg. Trends Eng. Res., vol. 8, no. 5, pp. 1497-1502, 2020. https://doi.org/10.30534/ijeter/2020/04852020

[9] R. Conejo, E. Guzmán, and M. Trella, "The SIETTE Automatic Assessment Environment," Int. J. Artif. Intell. Educ., vol. 26, no. 1, pp. 270-292, 2016. https://doi.org/10.1007/s40593015-0078-4

[10] D. E. Benchoff, M. P. Gonzalez, and C. R. Huapaya, "Personalization of Tests for Formative Self-Assessment,” Rev. Iberoam. Tecnol. del Aprendiz., vol. 13, no. 2, pp. 70-74, 2018. https://doi.org/10.1109/rita.2018.2831759

[11] Y. Fang, Z. Huanrui, and Y. Mingrui, "Enhancing english vocabulary learning via computerized adaptive testing," ICCSE 2017 - 12th Int. Conf. Comput. Sci. Educ., no. Iccse, pp. 35, 2017. https://doi.org/10.1109/iccse.2017.8085452

[12] F. E. Louhab, A. Bahnasse, and M. Talea, "Towards an Adaptive Formative Assessment in Context-Aware Mobile Learning,” Procedia Comput. Sci., vol. 135, pp. 441-448, 2018. https://doi.org/10.1016/j.procs.2018.08.195

[13] C. Sheng, B. Bingwei, and Z. Jiecheng, "An Adaptive Online Learning Testing System," in Proceedings of the 6th International Conference on Information and Education Technology, 2018. https://doi.org/10.1145/3178158.3178187

[14] H. T. Binh and B. T. Duy, "Student ability estimation based on IRT," in 2016 3rd National Foundation for Science and Technology Development Conference on Information and Computer Science (NICS), 2016, pp. 57-62. https://doi.org/10.1109/nics.2016.7725667

[15] L. S. Riza, R. A. Rosdiyana, A. Wahyudin, and A. R. Pérez, "The K-Means Algorithm for Generating Sets of Items in Educational Assessment," Indones. J. Sci. Technol., vol. 6, no. 1, pp. 93-100, 2021. https://doi.org/10.17509/ijost.v6i1.31523

[16] K. Chrysafiadi, C. Troussas, and M. Virvou, "Combination of fuzzy and cognitive theories for adaptive e-assessment,” Expert Syst. Appl., vol. 161, p. 113614, 2020. https://doi.org/10.1016/j.eswa.2020.113614

[17] A. Krouska, C. Troussas, and M. Virvou, "Computerized adaptive assessment using accumulative learning activities based on Revised Bloom's Taxonomy," in Smart Innovation, Systems and Technologies, 2019, vol. 108, pp. 252-258. https://doi.org/10.1007/978-3-31997679-2_26

[18] B. Jérôme, T. Hoffmann, R. Howard, W. Kraan, M. McKell, and C. Smythe, "IMS Question and Test Interoperability (QTI): Overview Version 2.2," 2015. https://www.imsglobal.org/question/qtiv2p2/imsqti_v2p2_oview.html (accessed Sep. 10, 2020).

[19] M. Boussakuk, A. Bouchboua, M. El Ghazi, M. El Bekkali, and M. Fattah, "Designing and Developing e-Assessment Delivery System Under IMS QTI ver.2.2 Specification,” Int. J. Emerg. Technol. Learn., vol. 16, no. 1, 2020. https://doi.org/10.3991/ijet.v16i01.16257

[20] G. T. Crisp, “Assessment in Next Generation Learning Spaces," in The Future of Learning and Teaching in Next Generation Learning Spaces, vol. 12, Emerald Group Publishing Limited, 2014, pp. 85-100. https://doi.org/10.1108/s1479-362820140000012009 
Paper-Design of Computerized Adaptive Testing Module into Our Dynamic Adaptive Hypermedia ...

[21] A. Abdelkader, D. Souilem, and R. Braham, "E-Assessment System Based on IMS QTI for the Arabic Grammar,” Int. J. Adv. Comput. Sci. Appl., vol. 5, no. 10, pp. 92-100, 2014. https://doi.org/10.14569/ijacsa.2014.051013

[22] G. Chemsi, M. Sadiq, M. Radid, and M. Talbi, "Formative E-assessment and behavioral commitment of students: Case of the Faculty of Science Ben M'sik,” Int. J. Emerg. Technol. Learn., vol. 14, no. 12, pp. 4-14, 2019. https://doi.org/10.3991/ijet.v14i12.10389

[23] N. Hrich, M. Lazaar, and M. Khaldi, "Problematic of the assessment activity within adaptive E-learning systems,” Int. J. Emerg. Technol. Learn., vol. 14, no. 17, pp. 133-142, 2019. https://doi.org/10.3991/ijet.v14i17.10675

[24] P. De Rizzo Meneghetti, Douglas Aquino Junior, "Application and simulation of computerized adaptive tests through the package catsim," ArXiv e-prints [stat.AP], 2017, [Online]. Available: http://arxiv.org/abs/1707.03012.

[25] X. Lan, H. Yu, and J. Li, "Optimization of Moodle for Adaptive Testing," in 2nd International Conference on Computational Modeling, Simulation and Applied Mathematics (CMSAM 2017), 2017, pp. 481-488.

[26] A. T. Nathan and J. W. David, "A Framework for the Development of Computerized Adaptive Tests,” Pract. Assessment, Res. Eval., vol. 16, no. 1, pp. 1-9, 2011. http://doi: 10.1108/ aaouj-01-2018-0009.

[27] A. H. Nabizadeh, J. P. Leal, H. N. Rafsanjani, and R. R. Shah, "Learning path personalization and recommendation methods: A survey of the state-of-the-art," Expert Syst. Appl., vol. 159, p. 113596, 2020. https://doi.org/10.1016/j.eswa.2020.113596

[28] K. Chrysafiadi and M. Virvou, "Student modeling approaches: A literature review for the last decade," Expert Syst. Appl., vol. 40, no. 11, pp. 4715-4729, 2013. https://doi.org/ 10.1016/j.eswa.2013.02.007

[29] N. Hrich, M. Lazaar, and M. Khaldi, "MAPSS: An intelligent architecture for the pedagogical support,” Int. J. Emerg. Technol. Learn., vol. 14, no. 14, pp. 19-30, 2019. https://doi.org/ 10.3991/ijet.v14i14.10362

\section{Authors}

Mohammed Boussakuk is a PhD student at the Laboratory of Artificial Intelligence, Data Sciences and Emerging Systems in Sidi Mohamed Ben Abdellah University (USMBA), Fez, Morocco. His major research interests are adaptive and intelligent tutoring systems, computerized adaptive testing and e-learning.

Ahmed Bouchboua received his PhD in Computer Science in 2017 from Sidi Mohamed Ben Abdellah University Abdellah (USMBA) Fez, Morocco. His major research interests are adaptive and intelligent tutoring systems, computerized adaptive testing and e-learning. (ahmed.bouchboua@usmba.ac.ma).

Mohammed El ghazi is a professor in the Electrical Engineering Department of Superior School of Technology at the Sidi Mohamed Ben Abdellah University (USMBA), Fez, Morocco. He is a member of Laboratory of Artificial Intelligence, Data Sciences and Emerging Systems. (mohammed.elghazi@usmba.ac.ma).

Moulhime El bekkali is a professor in the National School of Applied Sciences at the Sidi Mohamed Ben Abdellah University (USMBA), Fez, Morocco. He is a member of Laboratory of Artificial Intelligence, Data Sciences and Emerging Systems. (moulhime.elbekkali @usmba.ac.ma). 
Paper-Design of Computerized Adaptive Testing Module into Our Dynamic Adaptive Hypermedia ...

Mohammed Fattah received his Ph.D. in Telecommunications and CEM at the University of Sidi Mohamed Ben Abdellah (USMBA) Fez, Morocco, 2011. He is a professor in the Electrical Engineering Department of the High school of technology at the Moulay Ismail University (UMI), Meknes, Morocco and he is a responsible of the research team 'Intelligent Systems, Networks and Telecommunications', IMAGE laboratory, UMI. (fattahm@gmail.com).

Article submitted 2021-05-08. Resubmitted 2021-07-11. Final acceptance 2021-07-15. Final version published as submitted by the authors. 